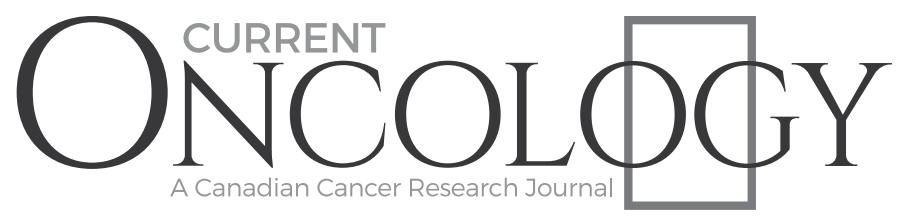

\title{
The Canadian Cancer Research Conference 2019
}

\author{
K.J.C. Galpin MSc, ${ }^{\text {ta }}$ D.P. Cook MSc, ${ }^{\text {ta }}$ L.M. Salemi PhD, ${ }^{\ddagger}$ S. Urowitz MSW PhD, ${ }^{\ddagger}$ \\ C. Williams PhD, $\$$ J.C. Bell PhD, ${ }^{\dagger \mid}$ M.D. Brundage MD MSc, ${ }^{* *}$ and B.C. Vanderhyden $\mathrm{PhD}^{* \dagger}$
}

\begin{abstract}
The 5th Canadian Cancer Research Conference (CCRC) took place 3-5 November 2019 in Ottawa, Ontario. Nearly 1000 participants - scientists, oncologists, community members, and patients-gathered to share knowledge, foster collaboration, and fuel the future of cancer research in Canada. The scientific program included 3 plenary sessions, 26 concurrent sessions, and 2 poster sessions presenting research described in more than 600 submitted abstracts, giving participants the opportunity to share health research that collectively encompassed the 4 pillars recognized by the Canadian Institutes of Health Research. In addition to the breadth of topics addressed by Canadian and international experts, the highlights of the meeting included the integration of patients and patient advocates, new rapid-fire sessions for abstract presentation, and events that enhanced learning opportunities for trainees.
\end{abstract}

Key Words Cancer research, patient engagement, meetings

Curr Oncol. 2020 April:27(2)e226-e230

www.current-oncology.com

\section{INTRODUCTION}

The Canadian Cancer Research Conference (CCRC) is a biennial meeting with these objectives:

Showcase the breadth and excellence of Canadian cancer research

1 Allow leading experts from across all areas of cancer research to exchange knowledge and share ideas to strengthen Canada's cancer research community

- Support new investigators in establishing themselves in the cancer research community

- Enhance patient involvement in cancer research in Canada

The event is organized by the Canadian Cancer Research Alliance (CCRA, https://www.ccra-acrc.ca/), an alliance of organizations that fund research into better ways to prevent, diagnose, and treat cancer and improve survivor outcomes ${ }^{1}$. Participants in the CCRC 2019 were drawn from across Canada, and the presentations addressed all 4 pillars of health research recognized by the Canadian Institutes of Health Research (CIHR, https://cihr-irsc.gc.ca/e/37788.html): biomedical research; clinical research; health services research; and social, cultural, environmental, and population health. In addition, all levels of conference organization undertook the necessary measures to strive for gender balance in all aspects of the conference. The Planning, Organizing, and Scientific committees achieved that balance through equitable representation on the committees and for session chairs and invited and selected-abstract speakers.

\section{MEETING REPORT}

\section{Advances and Discoveries in Cancer Research}

The CCRC strives to recognize and highlight the excellence of Canadian cancer research, spanning the 4 pillars of health research. All but 6 of the 122 oral presentations delivered throughout the course of the conference were therefore given by Canadian scientists. Plenary sessions were crafted in a unique way to bridge the pillars and facilitate cross-disciplinary discussion.

\section{Plenaries}

The opening conference plenary focused on novel cancer immunotherapies, with topics covering the fundamental biology of tumour immunology (Dr. Pamela Ohashi),

These authors contributed equally to the present work, with order determined by a coin flip. 
a Canadian-made clinical trial of a novel immunotherapy (Dr. Natasha Kekre), and the importance of cost-effectiveness analysis in the evaluation of new therapeutics (Dr. Jeffrey Hoch). The second plenary session discussed cancer genetics and precision oncology, which covered the importance of understanding the biology of genetic mutations in cancer, how that understanding can lead to personalized therapies (Dr. Jean-Yves Masson), and how shortcomings in our current system for genetic screening might prevent access to those treatments for patients who might derive benefit (Drs. Clare Turnbull and Kelly Metcalfe).

\section{Concurrent Sessions}

The concurrent sessions provided a more focused look into a variety of specific topics across the 4 pillars. Although space constraints prevent us from covering the impressive breadth of work, we highlight several broad topics from each pillar here.

\section{Immunotherapy}

Immunotherapy is a promising new class of cancer therapy that has been successful in the treatment of several cancers; however, for others, it provides little-to-no benefit ${ }^{1}$. Broadly, some cancer types are seemingly more resistant to therapy, and in many cancer types, immunotherapy offers benefit to only a subset of patients.

Many talks centred on understanding the biology of the tumour microenvironment and how the tumour cell types contribute to immunotherapy efficacy. Dr. Pamela Ohashi discussed novel immune cell subsets in tumours that modulate antitumour immunity and that might present new axes to inhibit therapeutically. Other talks defined the roles of nonimmune cells in the tumour microenvironment that promote tumour progression, including cancer-associated fibroblasts (Dr. Frédéric Pouliot) and astrocytes in brain metastases (Dr. Peter Siegel). The concurrent Cancer Immunotherapy session highlighted the variety of immunotherapeutic approaches currently used in the clinic or at the preclinical stage of development, including adoptive cell therapy, immune checkpoint blockade, and oncolytic virotherapy.

It is becoming increasingly apparent that the most effective strategy might be to design combination therapies to target tumours and the immune response from many angles $^{1,2}$. Doctoral student Ms. Alyssa Vito highlighted that concept by demonstrating that adding complementary cancer therapies can dramatically improve prognosis. Finally, the concurrent Model Systems in Cancer Research session, supported by talks outside the session, demonstrated the utility of model systems not only in studying the biology of cancer progression, but also in the rapid identification of novel therapeutic targets and testing the efficacy of new therapies.

\section{Personalized Therapies}

Much focus was also given to personalized therapies and to ensuring the identification of the patients that could benefit. Patients with several cancer types-including hormone-responsive breast tumours and $B R A F$-mutated melanoma-are already benefiting, but targeted therapies have yet to be developed for the greater proportion of tumours ${ }^{3}$. Improved screening for mutations in tumours will likely elucidate novel targets, but identifying nongenetic variations could expand the therapeutic possibilities.

Drs. Mathieu Lupien and Dalia Barsyte-Lovejoy discussed strategies for targeting epigenetic regulators in cancer cells; Dr. Josie Ursini-Siegel highlighted the benefits of targeting metabolic dependencies of the cells; and Dr. Francis Rodier suggested targeting senescence states in ovarian cancers. However, the development of new targeted therapies is beneficial only if the specific patients that could benefit can successfully be identified. Current genetic screening criteria miss patients who could benefit from screening, and existing screening panels fail to assess various mutations that could be actionable. Dr. Kelly Metcalfe reported that one third of $B R C A 1$ mutation carriers are not screened and discussed the merits of rapid screening at the time of diagnosis, emphasizing that early results can affect treatment planning and do not seem to have a negative psychological effect for patients. Such screens should also evolve as knowledge of biology expands with research. The PARP inhibitors have helped patients who have breast and ovarian cancers with BRCA1/2 mutations, but Dr. JeanYves Masson showed that those drugs could also benefit women with mutations in other enzymes associated with DNA repair, such as PALB2. Current panels fail to screen for those mutations, however.

\section{Clinical Trials}

Clinical trials also took centre stage at this year's conference. In the CCRC's first plenary session, Dr. Natasha Kekre discussed the first Canada-made trial for chimeric antigen receptor $T$ cell therapy. In addition to introducing a promising new therapy to Canadian patients, a key contribution of the trial has been the establishment of all the infrastructure required to manufacture the therapeutics and run the trials within Canada. Kekre emphasized the importance of this infrastructure for Canada in staying ahead of the curve, noting that this trial is only the first of Canadian-led immunotherapies in cancer.

Expanding on the importance of personalized medicine, Drs. Martin Smoragiewicz and James Rusthoven both discussed how clinical trials are identifying drugs for tumours of common histologic origins, but how advances in genomics have identified molecular stratifications of tumours for which most trials are not taking account. Dr. Tania Bubela discussed issues restricting innovation in clinical trials - namely, financial incentives that interfere with innovation and motivate "me too" therapeutics, often leveraging existing drugs for marginal improvements. She emphasized that research and innovation should not be restricted to the treatment itself, but should also shape regulatory, legal, and reimbursement frameworks.

\section{Prevention and Detection}

Although basic and clinical research are critical in the fight against cancer, improved strategies for prevention or detection at earlier stages would reduce the burden on patients and the health care system alike. For example, advances in screening programs have led to earlier detection of lung cancer (Dr. Martin Tammemagi), but inequities in access 
to education, employment, and income that are embedded in how society is organized has profound effects on health and health care access. Dr. Colleen Varcoe demonstrated the significant inequities concerning who is diagnosed with cancer and why, specifically related to differences in gender, Indigeneity, racialization, and income. Several initiatives have been established to address inequities in cancer screening in Canada.

Dr. Ambreen Sayani discussed recent work that is striving to identify barriers to lung cancer screening through interviews with high-risk individuals who declined cancer screening, with individuals who participated in screening, and with health care providers. Dr. Linda Rabeneck from Ontario Health (Cancer Care Ontario) highlighted innovative strategies for reducing the participation gap for screening, including a Facebook campaign that is leveraging the social media platform to target ads containing screening information to individuals based on geographic region.

Inequities in cancer prevention and screening faced by Indigenous populations were also a central topic of the conference. The World Indigenous Cancer Conference recently identified cancer in Indigenous populations as an urgent global concern. The cancer journey of Indigenous people is often characterized by barriers to accessing adequate health care, by other issues that lead to unmet needs, and by poorer outcomes compared with those in non-Indigenous Canadians.

A concurrent session this year focused entirely on Indigenous populations and cancer. The session featured talks from prominent experts about the breadth of the cultural perspectives of Indigenous patients and communities, and about how to improve care from the perspectives both of health care professionals and of community leaders. Dr. Angeline Letendre addressed Indigenous leadership in cancer care; Dr. Amanda Sheppard discussed improvements in cancer surveillance in Indigenous populations; and Dr. Charlotte Loppie advocated for transformative patient engagement.

Successful cancer prevention depends on an understanding of the exposures that contribute to cancer risk ${ }^{4}$. That need for understanding was highlighted and discussed in a variety of sessions. During a concurrent session dedicated to occupational cancer prevention, Dr. Cheryl Peters highlighted the burden in Canada of occupational cancers, which contribute thousands of cases each year, and the millions of Canadian citizens facing regular exposure to chemical and environmental workplace carcinogens. Dr. Vikki Ho discussed sex-specific differences in the cancers that can originate from exposure to endocrinedisrupting chemicals.

Drs. Christine Barul and Parveen Bhatti both discussed findings from recent studies examining overnight shift work as an occupational exposure that affects nearly 2 million Canadians. The International Agency for Research on Cancer has classified that exposure as a probable carcinogen. However, Bhatti discussed the methodology challenges that hinder an understanding of the exposure. Those challenges include gaps in the epidemiologic data (such as timing of the exposure relative to diagnosis) and failure to document the timing of biosample collectionfactors that are critical for an accurate assessment of the data. Developing strategies to effectively measure the exposure will be critical for understanding its involvement in cancer risk.

\section{Engaging Patient Advocates and the Community}

This year was the second that the CCRC included the Patient Involvement in Cancer Research Program (PIP), which helps to build the capacity of Canada's patient research advocates as part of the future of scientific research in Canada. The PIP participants co-chaired sessions, helped to lead discussions, and shared their personal experiences, which collectively aimed to inspire scientists and to put their research into broader context.

In one particular concurrent session, Stakeholder and Patient Engagement in Clinical Trials and Patient-Oriented Research, chaired by patient advocates Ms. Judy Needham and Ms. Lucy Piché, Needham gave an important talk about why and how patients should be involved in research. The patient perspective is important, and patients should be involved at all levels of scientific research: at the time the hypotheses are formulated, during data collection, and when conclusions are drawn and research is disseminated. In many contexts, patients are already involved in the granting process, in part to ensure inclusion of patientcentred protocols and endpoints.

In the same concurrent session, Dr. Caroline Hamm emphasized that patients receive the best care and attain the best outcomes when they are enrolled on a clinical trial, but in Ontario, only $7 \%$ of patients participate. The Clinical Trials Navigator was described as a tool for patients to use to identify which clinical trials they might be eligible for, thus giving patients the ability to help guide their own involvement in clinical trials. The aim is to shift the process away from centre- and location-based clinical research and to give more people opportunity to access care, which should generate stronger data and more knowledge. Importantly, those collaborations highlight the goal of involving patients in Canadian scientific research to help develop patient-centred treatments, outcomes, and research. Patient involvement helps to bridge the 4 pillars of research and helps scientists to think about their research from a multidisciplinary and patient-focused perspective.

To bring attention to the conference in the local community, the CCRA and its partners hosted, on 2 November 2019, a community event called Cancer: Piecing the Puzzle Together. Events were held in both French and English and included a keynote address by leading cancer researcher at The Ottawa Hospital Research Institute, Dr. John Bell, and Ms. Lucy van Oldenbarneveld, a breast cancer survivor and CBC News anchor. Bell discussed breakthroughs in cancer treatment and diagnoses in Canada, and van Oldenbarneveld shared her own story, as well as her thoughts about advances and the future of cancer research. Impossible to forget was Colorectal Cancer Canada's giant inflatable colon, which was both entertaining and educational about the signs, symptoms, and risk factors associated with colon cancer. That creative feature was a potent reminder of the screening programs in Canada that have the potential to save lives.

These programs all broadened awareness of current research and shared breakthroughs taking place in Canada. 
The anticipated outcome of bringing together the community, patients, families, and the public with scientists and their industrial partners is the creation of a strong Canadian scientific community committed to fighting cancer.

\section{Raising Up the Next Generation of Scientists}

New investigators gathered before the conference for an Early Career Investigator Program organized by the CIHR Institute of Cancer Research, the Ontario Institute for Cancer Research, and Prostate Cancer Canada, where they learned about the funding landscape from Drs. Christine Williams (Ontario Institute for Cancer Research) and Michael Strong (CIHR). They also heard from patient advocate Mr. Patrick Sullivan about the importance of involving patients from early points in the research development process and continuing to engage patients to guide the research. Furthermore, Sullivan advocated for new investigators to learn effective storytelling, so that they can share their exciting scientific discoveries not only with the scientific community, but also with the public, and so that they can use the power of their storytelling to inspire change.

Other satellite events included the Hack4Cancer Hackathon hosted by the Canadian Partnership Against Cancer, which brought trainees together to analyze cancer-linked data with the goal of shedding light on inequity in cancer care. The Science to Business Network held an event for trainees to learn about career opportunities in industry, government, and academia. The events were geared toward trainees, providing networking opportunities, career guidance, and ways to think about their roles in the scientific community from a multidisciplinary perspective.

In addition to the 60 invited speakers at CCRC 2019, more than 50 speakers were selected from among the submitters of more than 600 abstracts, giving opportunities to new investigators and trainees to share exciting, novel research. For the first time, the CCRC introduced "rapid-fire talks," increasingly considered a "gold standard" of effective, succinct communication. These thrilling 3-minute talks from all 4 pillars of health research gave young scientists a chance to excite the audience members about their research and to invite them to learn more during the poster sessions. The talks included promising new targeted therapies, immunotherapies, personalized medicine, prevention, and identification of risks-all serving to provide a quick snapshot of the breadth of research taking place across the country.

\section{Recognizing Canadian Excellence in Cancer Research} The CCRA recognizes individuals who have made exceptional contributions in terms of leadership, achievement, and service to cancer research. This year's recipient of the CCRA Award for Exceptional Leadership in Patient Involvement in Cancer Research was Mr. Patrick Sullivan, who gave an inspiring and moving speech about his son's experience with cancer, reminding researchers in the room of the importance of starting with "why" to make the "how" real and to help move cancer research forward faster and more efficiently.

The CCRA Award for Exceptional Leadership in Cancer Research is awarded to an individual who has made exceptional contributions to fostering the development of cancer research in Canada through their leadership in organizations or development of collaborative research opportunities or initiatives that have had a transformative effect. The winners this year were Dr. Anne-Marie Mes-Masson at the Institut du cancer de Montréal, Centre de recherche du Centre hospitalier de l'Université de Montréal, Université de Montréal, and Dr. Gerald Batist at McGill University, the Jewish General Hospital, the McGill Centre for Translational Research in Cancer, and the Montreal Centre for Experimental Therapeutics in Cancer. Mes-Masson received her award for establishing biorepositories for patient samples-work that has revolutionized translational medicine both in Canada and internationally. She shared her experience and wisdom with trainees and advocated giving back to the science ecosystem. Batist was recognized for the strides he has made in translating new discoveries from the lab to the clinic through multidisciplinary collaborations. He advocated for expanding our role as scientists in society and pointed to creating conversations with industry to advocate for lower drug costs and improved access to treatment. Both award winners emphasized the importance of collaborations to accelerate scientific research, but also to improve public awareness and engagement of patients.

The CCRA Award for Outstanding Achievements in Cancer Research recognizes outstanding lifetime achievements that have greatly advanced the understanding of cancer, the treatment of cancer, or cancer control. This year's award was presented to Dr. Lincoln D. Stein of the Ontario Institute for Cancer Research, University of Toronto, and Cold Spring Harbor Laboratory, for his work in pioneering data sharing. Through his efforts, datasets are shareable and accessible, leading to responsible global sharing of cancer genome data to advance knowledge and pool wisdom for better disease management.

The CCRA Award for Distinguished Service to Cancer Research is awarded to someone whose work has had a substantial impact on public education, policy, or discourse in cancer control and particularly cancer research in Canada. This year's recipient was Dr. Geoffrey T. Fong at the University of Waterloo and Ontario Institute for Cancer Research for his work in the creation of the International Tobacco Control policy evaluation project, an advocacy project leading to the prevention of millions of tobacco-related cancer deaths in Canada.

\section{The Future of Cancer Research in Canada}

The CCRC closed with a plenary session dedicated to discussing the future of cancer research in Canada. The session served as an opportunity for Dr. Stephen Robbins (CIHR) to introduce the draft of the pan-Canadian Vision for Cancer Research: "Every person in Canada is part of a bold movement to push the frontiers of cancer research and translate all promising discoveries into maximum health and wellbeing."

Beyond simply promoting innovation, this vision highlights Canada's value that research is an endeavour taken on not just by scientists, but by all Canadian citizens. This year's CCRC scientific program demonstrated that the country is already making strides toward that goal: the PIP demonstrated the power of patient involvement in research; the first Canadian-led clinical trial for immunotherapies 
in Canada was established and is underway, removing global dependencies; and active conversations are in progress about how to innovate in realms beyond biology, from the economics of clinical trials to challenging inequities in cancer diagnosis and treatment. The outcomes of those conversations will undoubtedly lead to interventions that significantly reduce cancer risk and cancer burden across all populations.

\section{ACKNOWLEDGMENTS}

Thank you to all members of the 2019 CCRC Executive Planning Committee, Scientific Program Committee, and Local Organizing Committee. The CCRC was supported by 20 CCRA member organizations, 8 academic departments and research institutes, 5 industry partners, 4 hospital foundations, and 4 government and nongovernmental organizations. For a full list of committee members and supporters please download the CCRC 2019 scientific program at https://conference.ccra-acrc.ca/past-conferences/2019-ccrc/.

\section{CONFLICT OF INTEREST DISCLOSURES}

We have read and understood Current Oncology's policy on disclosing conflicts of interest, and we declare that we have none relevant to this report.

\section{AUTHOR AFFILIATIONS}

*Department of Cellular and Molecular Medicine, University of Ottawa, Ottawa; ${ }^{\dagger}$ Cancer Therapeutics Program, The Ottawa Hospital Research Institute, Ottawa; ${ }^{\ddagger}$ Canadian Cancer Research Alliance, Canadian Partnership Against Cancer, Toronto; ${ }^{\S}$ Ontario Institute for Cancer Research, Toronto; $\|$ Department of Biochemistry, Microbiology and Immunology, University of Ottawa, Ottawa; \#Queen's Cancer Research Institute, Department of Oncology, Queen's University, Kingston, ON.

\section{REFERENCES}

1. Kruger S, Ilmer M, Kobold S, et al. Advances in cancer immunotherapy 2019-latest trends. J Exp Clin Cancer Res 2019; 38:268.

2. Galon J, Bruni D. Approaches to treat immune hot, altered and cold tumours with combination immunotherapies. Nat Rev Drug Discov 2019;18:197-218.

3. Chae YK, Pan AP, Davis AA, et al. Path toward precision oncology: review of targeted therapy studies and tools to aid in defining "actionability" of a molecular lesion and patient management support. Mol Cancer Ther 2017;16:2645-55.

4. Loomans-Kropp HA, Umar A. Cancer prevention and screening: the next step in the era of precision medicine. NPJ Precision Oncology 2019;3:3. 